\title{
Non-gravitational forces and masses of some long-period comets. The cases of Hale-Bopp and Hyakutake
}

\author{
Andrea Sosa and Julio A. Fernández \\ Departamento de Astronomía, Facultad de Ciencias, \\ Igua 4225, 11400, Montevideo, Uruguay \\ email: asosa@fisica.edu.uy, julio@fisica.edu.uy
}

\begin{abstract}
By means of a simple non-gravitational force model of the cometary nucleus, which relies on the observed light curves assumed to be a good representation of the water sublimation rate, we estimate the masses of a sample of long-period comets (LPCs).

A critical issue of our method is the assumption of a correlation between visual heliocentric magnitudes and water production rates. This is a necessary assumption because of the sparse observational data of gas production rates (with the exception of very few comets like Hale-Bopp or Hyakutake). In this regard we present here a new correlation for LPCs. We also present the preliminary results for the masses of comets Hale-Bopp and Hyakutake.
\end{abstract}

Keywords. Comets: general, methods: analytical, data analysis.

\section{Introduction}

The main non-gravitational effect that can be detected in a periodic comet is a change in its orbital period, with respect to that derived from purely gravitational theory. Whipple (1950) proposed an icy conglomerate model for the cometary nuclei, and showed that the momentum transferred to the nucleus by the outgassing could cause the observed non-gravitational effect.

A method for computing cometary masses based on the non-gravitational effect was introduced by Rickman (1986, 1989), Rickman et al. (1987), and Sagdeev et al. (1987). Similar approaches were presented later by Rickman et al. (1991), and more recently by Szutowicz et al. (2002a, 2002b), Farnham \& Cochran (2002), Szutowicz \& Rickman (2006), and Sosa \& Fernández (2009).

Most of the studies of comet masses are for Jupiter family comets (JFCs). Unlike the short-period comets, the evaluation of non-gravitational forces is more difficult for LPCs, since these have not been observed in a second apparition to check for a change in the orbital period attributable to non-gravitational forces. Nevertheless, non-gravitational terms have been fitted to the equations of motion of several LPCs leading to more satisfactory orbital solutions.

A total of 30 comets, observed between 1973 and 2007, have been used in our study, selected from the known population of LPCs with orbital periods $\mathrm{P}>1000 \mathrm{yr}$ and short perihelion distances $(\mathrm{q}<2 \mathrm{AU})$, by demanding that each comet has measured water production rates and a data set of photometric observations good enough to determine a light curve. We use the visual magnitudes from the International Comet Quaterly database, while the gas production data are collected from the literature and other public sources, like the Nançay database of $\mathrm{OH}$ production rates, and the International Astronomical Union Circulars. The orbital and non-gravitational parameters are extracted from the Catalogue of Cometary Orbits (Marsden \& Williams 2008). 


\section{The method}

According to Whipple (1950), we can relate the non-gravitational acceleration $\vec{J}$ and the comet's mass $M$ by means of the conservation of momentum i.e.

$$
M \vec{J}=-Q m \vec{u}
$$

where $Q$ is the gas production rate, $\vec{u}$ is the effective outflow velocity, and $m$ is the average molecular mass.

The non-gravitational acceleration (which is in the opposite direction to that of the net outgassing) can be described in terms of its radial (i.e. in the antisolar direction), transverse (i.e. perpendicular to the radial component in the direction of the motion), and normal (i.e. perpendicular to the orbital plane) components $\vec{J}_{r}, \vec{J}_{t}$ and $\overrightarrow{J_{n}}$, respectively. According to the standard symmetric model developed by Marsden et al. (1973), we have $J_{r}=A_{1} g, J_{t}=A_{2} g$, where the parameters $A_{1}, A_{2}$ represent the radial and transverse components of the non-gravitational acceleration at 1 AU from the Sun, respectively, and $g=g(r)$ is an empirical function which describes the variation of the water snow sublimation rate with respect to the heliocentric distance. The NG parameters $\left(A_{1}, A_{2}\right)$ arise from the best-fitting orbital solution.

Finally, by taking absolutes values for the vectors in eq. (2.1), and solving for the mass, we derive the following expression:

$$
M=\frac{Q m<u>}{J}
$$

where $J=\sqrt{A_{1}^{2}+A_{2}^{2}} \times g$, and $\langle u>$ represents an average value for all escaping molecules during the passage of the comet in the inner solar system $(r<3 \mathrm{AU})$. As an educated guess, we chose $\langle u\rangle=(0.27 \pm 0.1) \mathrm{km} \mathrm{s}^{-1}$ (see Sosa \& Fernández 2009 for further details). At heliocentric distances $r<3 \mathrm{AU}$ the cometary activity is governed by the sublimation of water ice, so we take $m$ as the water molecular mass. As we can see from eq. (2.2), the computation of the cometary mass requires to know from observations the shape of the curve $Q$. Due to the scarce measurements of gas production rates we assume an empirical law introduced by Festou (1986), to approximately convert total visual heliocentric magnitudes $m_{h}$ (i.e. the apparent magnitudes $m$ corrected for the geocentric distance $\left.\Delta: m_{h}=m-5 \log \Delta\right)$ into water production rates,

$$
\log Q=a_{1} \times m_{h}+a_{0}
$$

The light curve $m_{h}(\mathrm{t})$ is defined by a polynomial fit to the upper envelope of the ensemble of photometric observations (details of the procedure can be found in Sosa \& Fernández 2009).

\section{The results and concluding remarks}

Fig. 1 shows the light curves obtained as plots of the total heliocentric visual magnitude as a function of the time relative to the perihelion passage, for comets Hale-Bopp and Hyakutake. We performed a linear regression between the heliocentric magnitudes (as inferred from the light curves), and the measured water production rates, by an iterative procedure that removes the observations outside $3-\sigma$ of the residuals. We obtained a sample of 585 data points for $30 \mathrm{LP}$ comets, for which the heliocentric distances varied between 0.20 and $2.98 \mathrm{AU}$, while the geocentric distances spanned from to 0.10 to 3.04 

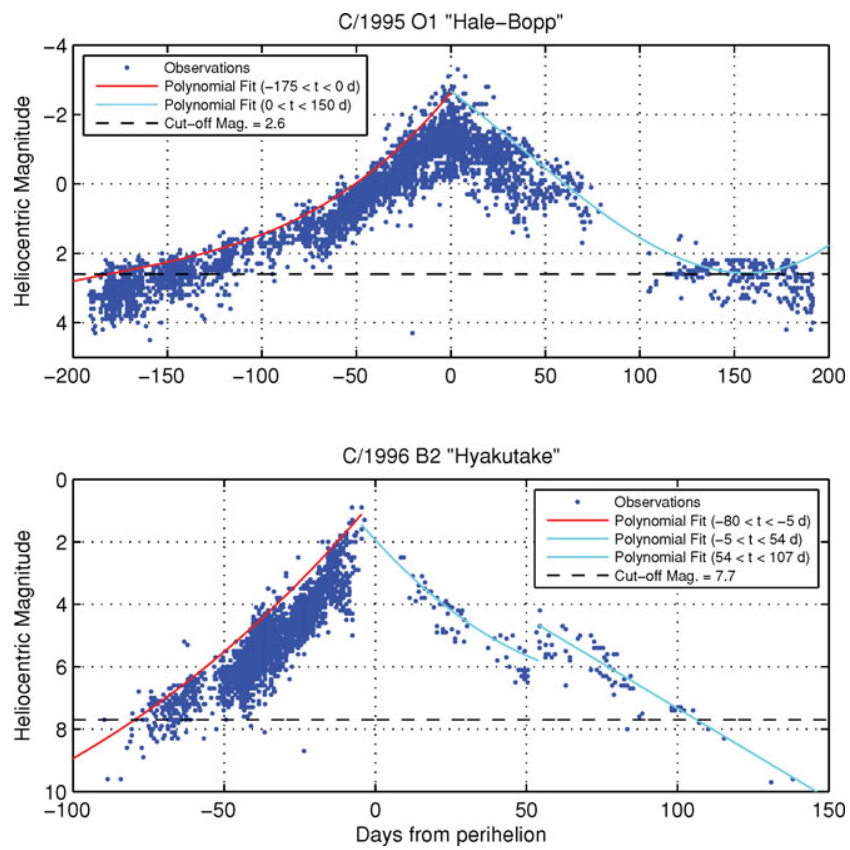

Figure 1. Heliocentric total visual magnitudes as a function of time, for comets Hale-Bopp (up) and Hyakutake (bottom). The polynomial fit $m_{h}(t)$ to the upper envelope of the broad distribution of photometric measurements is shown. The horizontal line indicate the cut-off magnitude $m_{h C}$. A total of 4576 and 3101 observations were used for HB and Hyakutake, respectively.

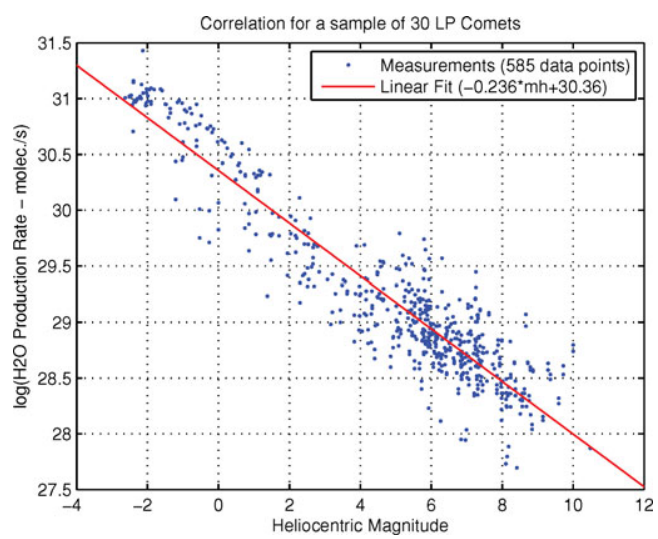

Figure 2. The points indicate the data (logarithm of the measured water production rate vs. the estimated visual heliocentric magnitude from the light curves), while the line shows the correlation law.

AU. For this sample, the best fitted coefficients were $a_{1}=-0.236, a_{0}=30.26$, with a regression coefficient of -0.94 (Fig. 2).

With our work still in progress, we present here only the preliminary results for comets Hale-Bopp and Hyakutake. We note that the shape of $Q(r)$ differs from $g(r)$, as it is evidenced by the light curves of Fig. 1, which show certain degree of asymmetry with respect to the perihelion. Hence, we will have different values of $M$, as $r$ varies. Since we are 
interested in an average value for $M$ (neglecting the loss of mass due to the outgassing), we consider the variations in $M$ due to such difference as a source of uncertainty inherent to the method; the more the ratio $Q / g$ approaches unity, the more constrained will be the range of possible values for $M$. Our preliminary results lead to a mass estimate within the range $\sim[1.6-2.7] \times 10^{14} \mathrm{~kg}$ for comet Hale-Bopp , while the mass estimate for comet Hyakutake would be within the range $\sim[1.3-3.3] \times 10^{12} \mathrm{~kg}$.

We find a strong correlation between visual magnitudes and water production rates, like previous works. Our results are similar to that of Jorda et al. (2008), practically with the same slope (they derived $a_{1}=-0.2453$ ), but with a slightly smaller value for $a_{0}$ (they derived $a_{0}=30.675$ ), possibly due to the method chosen to derive the visual magnitudes (e.g. we privilege the brightest observations over the faintest, while Jorda et al. average the observations). Other main differences with respect to the work of Jorda et al. (besides the method for determining the visual magnitudes) are that they consider only the Nançay database (with a smaller sample size), and that they did not distinguish between dynamical classes, while we consider all water measurements (obtained by the different techniques), and restrict our study to LPCs. As future work, it remains a more complete statistical analysis of the correlation, by including the error estimates.

The mass estimates for comets Hale-Bopp and Hyakutake are in good agreement with previous works of Szutowicz et al. (2002a) and Szutowicz et al. (2002b), respectively. In comparison with the masses derived by us (Sosa \& Fernández 2009) for a sample of JFCs, comet H-B would be a "massive" comet, belonging to the same mass rank as comets Halley and Tempel 2, while comet Hyakutake would be a "lighter" comet, in the same mass rank as comets d'Arrest and Borrelly.

\section{References}

Farnham, T. L. \& Cochran, A. L. 2002, Icarus, 160, 398

Festou, M. 1986, in: Proc. Asteroids, Comets, Meteors 1985

Jorda, L., Crovisier, J., \& Green, D. W. E. 2008, in: Proc. Asteroids, Comets, Meteors 2008

Marsden, B. G., Sekanina, Z., \& Yeomans, D. K. 1973, AJ, 78, 211

Marsden, B. G. \& Williams, G. V. 2008, Catalogue of Cometary Orbits, 17th ed., Smithsonian Astrophysical Observatory

Rickman, H. 1986, in: ESA SP 249

Rickman, H. 1989, Adv. Sp. Res., 9, 59

Rickman, H., Kamél, L., Festou M., \& Froeschlé, C. 1987, in: ESA SP 278

Rickman, H., Festou, M. C., Tancredi, G., \& Kamél, L. 1991, in: Proc. Asteroids, Comets, Meteors 1991, p. 509

Sagdeev, R. Z., Elyasberg, P. E., \& Moroz, V. L. 1987, Sov. Astron. Lett., 13, 259

Sosa, A. \& Fernández, J. A. 2009, MNRAS, 393, 192

Szutowicz, S. \& Rickman, H. 2006, Icarus, 185, 223

Szutowicz, S., Królikowska, M., \& Sitarski, G. 2002a, Earth, Moon and Planets, 90, 119

Szutowicz, S., Królikowska, M., \& Sitarski, G. 2002b, in: Proc. Asteroids, Comets, Meteors 2002

Whipple, F. L. 1950, AJ, 111, 375 